\title{
Composition and Configuration Dependence
} of Glass Transition Temperature in Binary Copolymers and Blends of

\section{Polyhydroxyalkanoate Biopolymers}

Karteek K. Bejagam, ${ }^{* \dagger}$ Carl N. Iverson, ${ }^{\ddagger}$ Babetta L. Marrone, $₫$ and Ghanshyam

$$
\text { Pilania*, }
$$

$\dagger$ †aterials Science and Technology Division, Los Alamos National Laboratory, Los Alamos, $N M 87545, U S A$

$\ddagger$ Chemistry Division, Los Alamos National Laboratory, Los Alamos, NM 87545, USA

9Bioscience Division, Los Alamos National Laboratory, Los Alamos, NM 87545, USA

E-mail: karteekbeja@lanl.gov gpilania@lanl.gov 


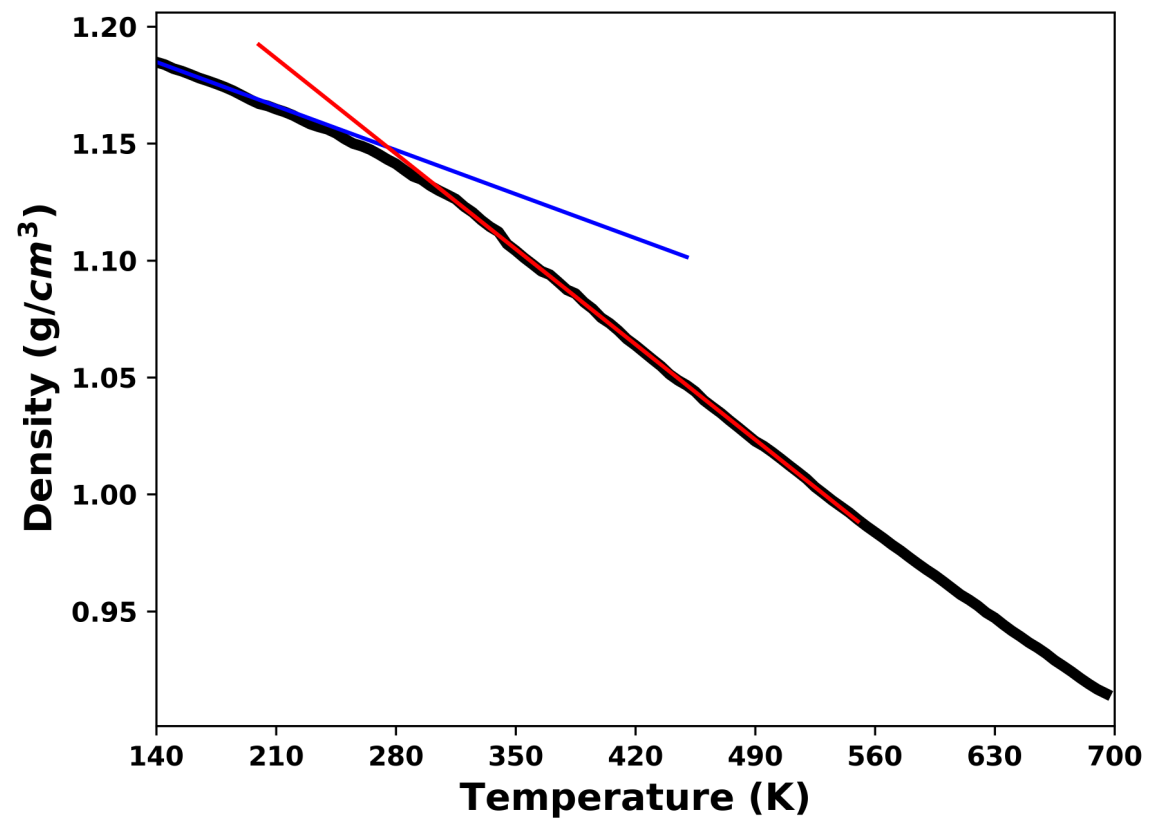

Figure S1: Density as a function of temperature obtained from the quenched simulations of random copolymer of $\mathrm{P} 3 \mathrm{HB}-\mathrm{P} 4 \mathrm{HB}$ at $10 \mathrm{~mol} \%$ of $\mathrm{P} 4 \mathrm{HB}$. 

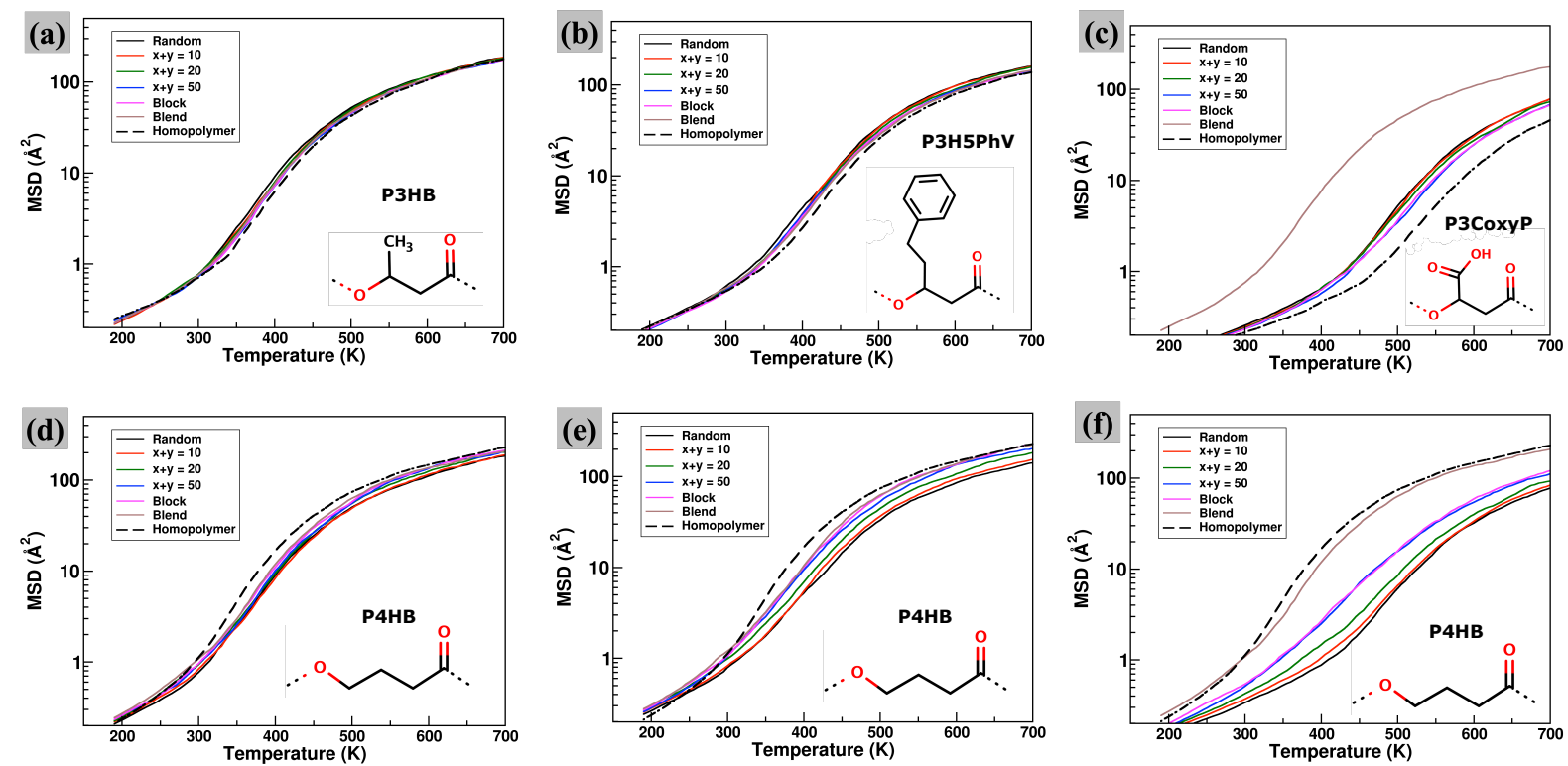

Figure S2: Configuration-dependent monomer mean-square displacement versus temperature curves for monomers of (a) 3HB in P4HB-P3HB, (b) 3H5PhV in P4HB-P3H5PhV, (c) 3CoxyP in P4HB-P3CoxyP, (d) 4HB in P4HB-P3HB (e), 4HB in P4HB-P3H5PhV and (f) $4 \mathrm{HB}$ in $\mathrm{P} 4 \mathrm{HB}-\mathrm{P} 3 \mathrm{CoxyP}$. The black dashed lines show the homopolymer mean-square displacement profiles of the monomer depicted in the respective panels for a direct comparison. All the results for binary polymer systems are computed at a composition of $30 \mathrm{~mol} \%$ of P4HB.
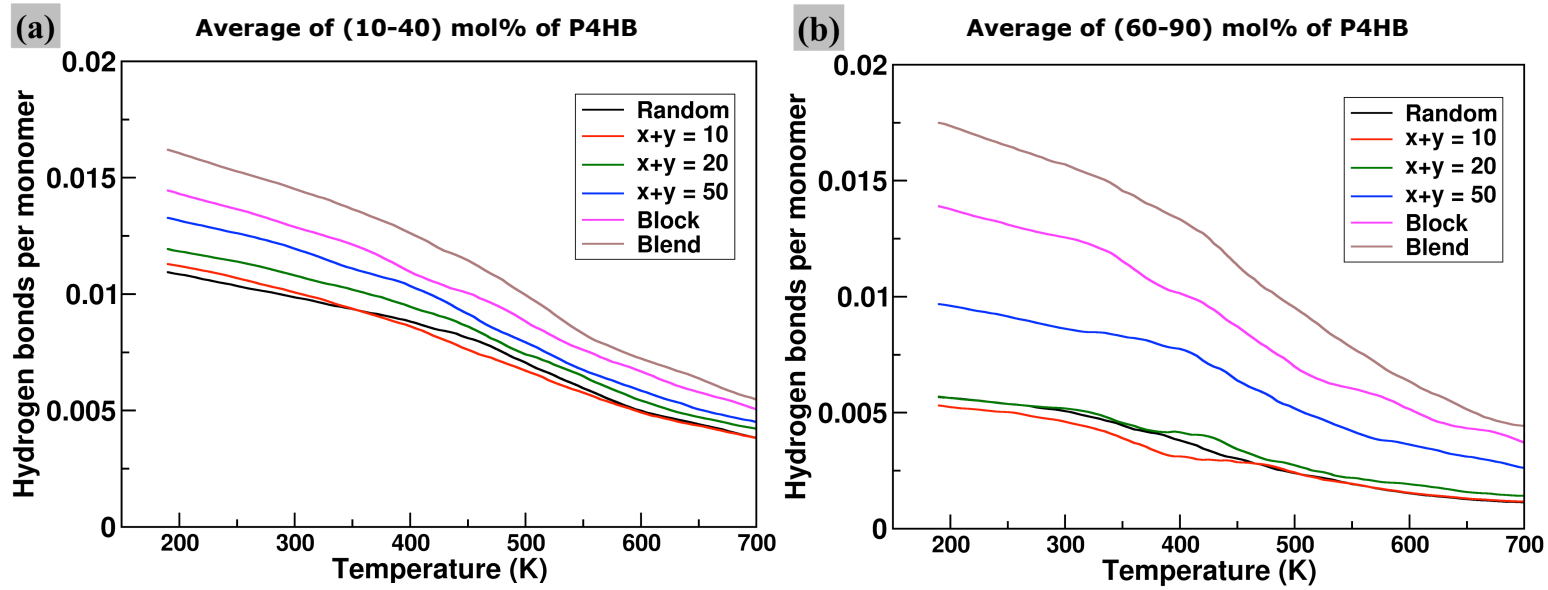

Figure S3: Number of hydrogen bonds normalized per 3CoxyP monomer in different polymeric configurations of the binary $\mathrm{P} 4 \mathrm{HB}-\mathrm{P} 3 \mathrm{CoxyP}$ system averaged over composition ranges of (a) 10-40 mol \% and (b) 60-90 mol\% of P4HB, respectively. 


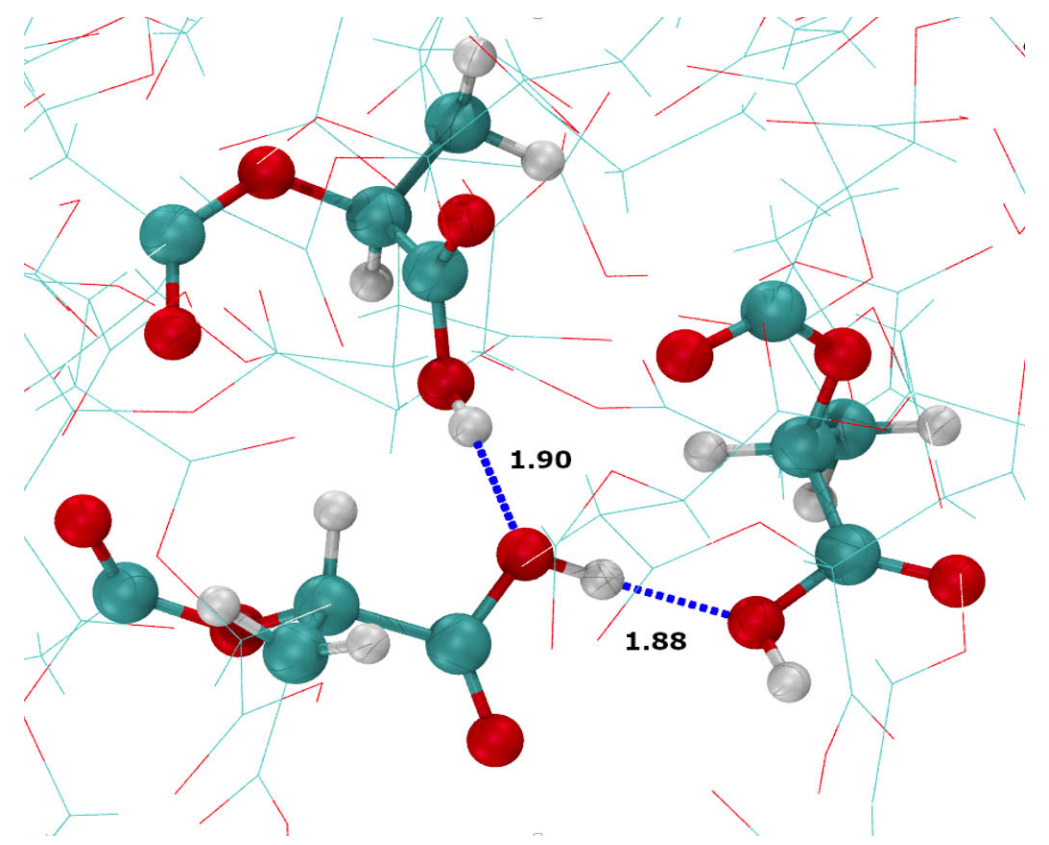

Figure S4: A representative snapshot illustrating the presence of hydrogen bonds between the carboxylate group of $3 \mathrm{Coxy} \mathrm{P}$ monomers. All the polymer chains are represented with a stick model and only the monomers involved in hydrogen bonding are highlighted. Distance between the acceptor and hydrogen is shown in $\AA$ units. 